\section{Building blocks}

Focal adhesions are multiprotein complexes that mediate interactions between cells and the extracellular matrix. They have both a mechanical, scaffolding role and a signaling one. Previous electron microscopy studies revealed that focal adhesions have an

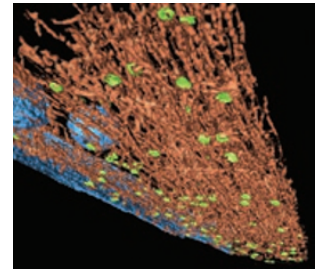

electron-dense cytoplasmic layer with cytoskeletal proteins associated, but their three-dimensional organization was not known. Patla et al. have now combined fluorescence microscopy and cryoelectron tomography to uncover the molecular architecture of focal adhesions. At the heart of the focal adhesions, where the actin fibers meet the plasma membrane, they found hundreds of heterogeneous doughnut-shaped particles with diameters of between 20 and $30 \mathrm{~nm}$, separated by $43 \mathrm{~nm}$ on average. These focal adhesion-associated particles seem to provide 'building blocks': that is, instead of having a central focal adhesion component, the particles likely provide flexibility, by assembling and disassembling in response to environmental conditions, and this allows focal adhesions to adapt to stimuli such as mechanical force. For example, in response to inhibition of Rho kinase, the size of these particles shrinks rapidly, suggesting a link to mechanosensing. The overall morphology of these complexes changes in response to contractile forces, such as those resulting from inhibition of Rho kinase. Focal adhesions are composed of four main structural domains; the outermost domain is the plasma membrane, with integrin receptors to anchor the membrane to the extracellular matrix, and the focal adhesionassociated particles are located at the cytoplasmic face and interact with short fibers that are associated with actin stress fibers. This new insight into the modular nature of focal adhesions hints at how these structures can link the cell's cytoskeleton to the extracellular matrix. (Nat. Cell Biol. doi:10.1038/ncb2095, published online 8 August 2010)

$\mathrm{MH}$

\section{Lagging preference}

Although many transposons employ a nonreplicative, cut-and-paste mechanism to move to a new location, a recently described family of transposons, typified by IS608, uses a unique mechanism involving a single-stranded DNA intermediate. IS608's transposase (TnpA) cleaves the top strand of the element, excising a single-stranded circle that inserts only into single-stranded target DNA, at the $3^{\prime}$ side of the sequence TTAC. The source of the single-stranded target DNA was unclear, and one possibility was that it was generated during replication. Chandler and colleagues now find that IS608 excision from a replicating plasmid is observed only when the top strand of the transposon is in the lagging strand. Disruption of the replication fork to increase the amount of ssDNA enhanced excision. By manipulating the frequency of Okazaki fragment initiation to change the length of ssDNA, they found a correlation between the length of the ssDNA and the size of the transposon resulting in most efficient excision, although transposons longer than the ssDNA show residual activity whose basis remains to be determined. IS608 insertion into the bacterial chromosome also shows a strong preference for the lagging strand. When a replication fork is stalled at a defined site, insertion is still seen into the lagging strand at the end of the TTAC sequence. The genomic locations of these elements in several bacterial species confirm that their insertion was highly biased to the lagging strand. Although other transposons, such as Tn7, show a strand preference for insertion and use replication forks, they act via dsDNA intermediates. Therefore, IS608 illustrates how different mechanisms can make use of a common process. (Cell 142, 398-408, 2010)

$A K E$

\section{Repair signal}

Replication protein A (RPA) binds to single-stranded DNA in response to replication stress, coating the damaged DNA to remove any secondary structure. RPA is required for repair of double-strand break repair through homologous recombination, and it has a key role in recruiting the recombinase Rad51. How RPA signals to Rad51 was not clear, but Dou et al. show that SUMO plays a key part in regulating DNA repair. RPA is composed of three subunits: RPA70, RPA32 and RPA14. The SUMO-specific protease SENP6 associates with RPA70 in the nucleus and maintains RPA in a hypoSUMOylated state during S phase. Upon replication stress induced by camptothecin or ionizing radiation, SENP6 dissociates from RPA70, and RPA70 is then modified by SUMO $2 / 3$ on lysine residues 449 and 577 . This induces recruitment of Rad51 to the DNA damage site to start repair through homologous recombination. Mutations in RPA that prevent SUMOylation also result in defective homologous recombination and confer higher sensitivity to camptothecin. Thus, SUMOylation of RPA70 is crucial for regulation of DNA repair through homologous recombination. (Mol. Cell 39, 333-345) MH

\section{Hidden channel}

$\mathrm{Na}^{+} / \mathrm{K}^{+}$ATPases are involved in a number of key processes in animal cells, including ion transport and signaling events, and numerous mutations have been linked to neurological disorders. Previous crystal structures and functional analyses have given insight into the movement of ions, which is asymmetric and involves passage of three sodium ions out of and two potassium ions into the cell for each ATP used. Such previous analyses defined the ion paths and key ion-binding sites, but now Poulsen, Nissen and colleagues have found an unexpected additional ion pathway. Whereas two of the previously known ion-binding sites are conserved between $\mathrm{Na}^{+} / \mathrm{K}^{+}$ATPases and the sarcoendoplasmic $\mathrm{Ca}^{2+}$ ATPase, the unique third sodium site has been proposed to lie between transmembrane helices (TM) 5, 8 and 9. Functional studies have linked the third site to a C-terminal structure in the $\mathrm{Na}^{+} / \mathrm{K}^{+}$ATPase. The crystal structure and mutational studies suggested an alternative third binding site. Using mutants in the $\mathrm{C}$-terminal region that were identified in familial hemiplegic migraine 2 (FHM2), linked to a dominantly inherited severe form of migraine, the authors carried out electrophysiological studies that argue that this region is involved in regulating the gate to an ion pathway. Furthermore, a buried aspartic acid residue is a key constituent of this C-terminal pathway and is found mutated to asparagine in a severe form of rapid-onset dystonia with parkinsonism (RDP). The mutational analysis indicates that the disengagement of the $\mathrm{C}$-terminal region from the transmembrane domain is the rate-limiting step for sodium release. Molecular dynamics simulations support the idea that the polar side chain-lined region between the $\mathrm{C}$-terminal domain and the third ion-binding site can be occupied by water. The analysis further suggests that the proposed new ion pathway is likely to be conserved in related pumps, and the mechanism of ion passage proposed as a result of these findings fulfills the criteria of the "4-site model", suggested over 30 years ago as important for the catalytic mechanism of ion transport. The study is therefore consistent with a body of previous data but also uses disease-associated alleles to understand the mechanism of ion transport and at the same time suggests a basis for the defect that may underlie neurological diseases. (Nature, doi:10.1038/ nature09309, published online 15 August 2010) $S L$ 